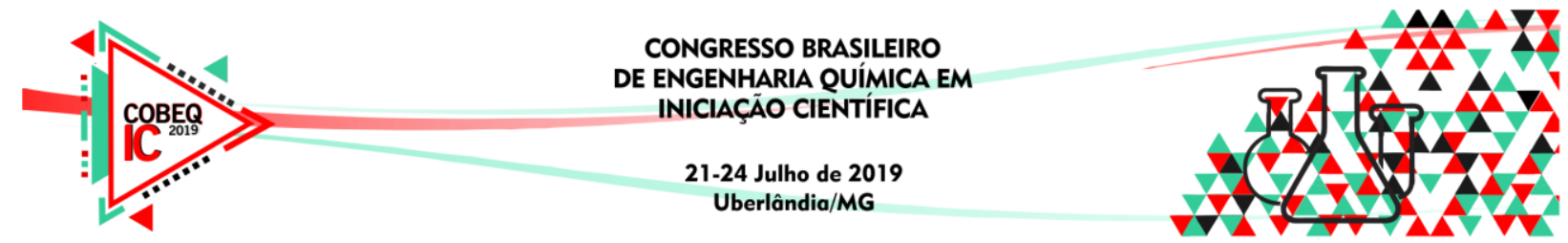

\title{
FERMENTAÇÃO DE SORO LÁCTEO E ÁGUA DE MACERAÇÃO DE MILHO UTILIZANDO CULTURA MISTA DE Propionibacterium acidipropionici E Lactobacillus paracasei
}

\author{
C. C. O. P. TOURINO ${ }^{1}$; B. I. SERPA ${ }^{2}$, M. T. NGOME ${ }^{3}$; O. L. MONDRAGÓN-BERNAL ${ }^{4}$; J. \\ G. L. F. ALVES ${ }^{5}$ \\ ${ }^{1}$ Universidade Federal de Lavras, Departamento de Engenharia \\ ${ }^{2}$ Universidade Federal de Lavras, Departamento de Engenharia \\ ${ }^{3}$ Universidade Eduardo Mondlane, Departamento de Produção Agrícola \\ ${ }^{4}$ Universidade Federal de Lavras, Departamento de Ciência dos Alimentos \\ ${ }^{5}$ Universidade Federal de Lavras, Departamento de Ciência dos Alimentos \\ E-mail para contato: ctourino@estudante.ufla.br, jlembi@ufla.br
}

\begin{abstract}
RESUMO - O ácido propiônico (AP) possui muitas aplicações industriais, porém atualmente é produzido por síntese química a partir de derivados do petróleo, com geração de resíduos muito poluentes. Uma alternativa para obtenção desse ácido é a via fermentativa, utilizando bactérias propiônicas e subprodutos agroindustriais. Com o objetivo de avaliar a produção de AP a partir do soro de leite concentrado, água de maceração de milho (AMM) e carbonato de cálcio $\left(\mathrm{CaCO}_{3}\right)$ por cultura mista de Propionibacterium acidipropionici e Lactobacillus paracasei, foi realizado um delineamento composto central rotacional (DCCR). Foram feitos 12 tratamentos, em triplicata, utilizando diferentes concentrações de lactose de soro lácteo e AMM, mantendo uma concentração fixa de $40 \mathrm{~g} / \mathrm{L}$ de $\mathrm{CaCO}_{3}$. O processo fermentativo ocorreu por $120 \mathrm{~h}$ e foram retiradas amostras dos tratamentos a cada 24h para realização posterior de análises cromatográficas e no tempo inicial e final para contagem celular. Observou-se que a concentração de lactose e AMM exerceram efeitos positivos estatisticamente significativos sobre produção do AP, sendo o tratamento com concentração de lactose de $40 \mathrm{~g} / \mathrm{L}$ e concentração de AMM de $65 \mathrm{~g} / \mathrm{L}$ o que apresentou a maior produção de AP $(6,84 \mathrm{~g} / \mathrm{L})$. Pode-se concluir dos resultados que concentrações de lactose e AMM acima de $45 \mathrm{~g} / \mathrm{L}$ e de $50 \mathrm{~g} / \mathrm{L}$ nos meios de fermentação, respectivamente, favorecem a produção de AP.
\end{abstract}

\section{INTRODUÇÃO}

O ácido propiônico é um importante produto químico intermediário na síntese da vitamina E, fibras de celulose, aromas artificiais de frutos, fragrâncias, perfumes e os seus sais são amplamente utilizados na conservação de alimentos e rações. É também um interessante intermediário na produção de polímeros, plásticos, pesticidas, produtos farmacêuticos, solventes e bioaromas (CHEN et al., 2012). Atualmente o ácido propiônico é produzido por síntese química de derivado do petróleo (DISHISHA et al., 2012). Os combustíveis fósseis são economicamente mais atraentes do que vetores energéticos alternativos, entretanto, há um interesse crescente na viabilidade econômica e na produção sustentável de produtos químicos a partir de recursos renováveis. Uma via alternativa que está sendo extensivamente 


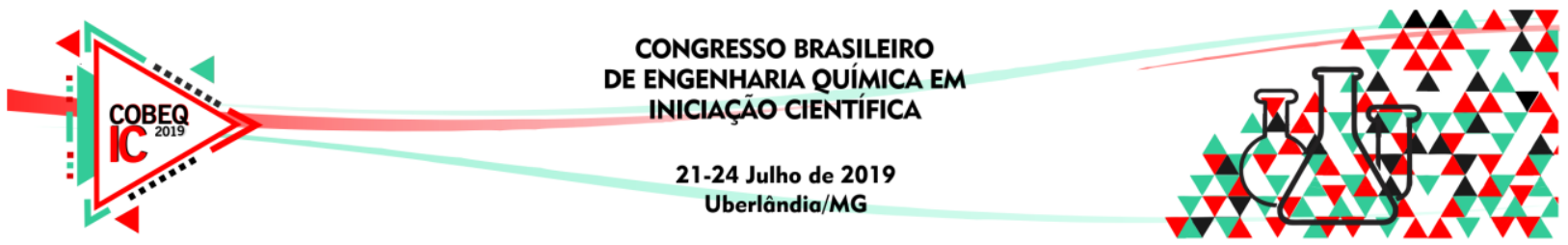

investigada é a produção fermentativa de ácido propiônico a partir de recursos baratos e renováveis, que possui grande interesse por não gerar muitos resíduos, o que caracteriza esse sistema de produção como verde ou sustentável (SABRA et al., 2013), além de apresentar a possibilidade de utilização de matérias-primas baratas, geralmente resíduos industriais, com grande disponibilidade no mercado.

O soro lácteo resultante da produção de queijos é interessante para o processo fermentativo, tanto em função da sua elevada produção, quanto à rica composição nutricional, porque apresenta mais da metade dos sólidos, incluindo grande parte da lactose, cerca de 20 $\%$ das proteínas, vitaminas e sais minerais presentes no leite original (ATRA et al., 2005; BALDASSO et al., 2011). Outro subproduto de interesse é resultante do processo de beneficiamento de milho por via úmida, chamado de milhocina ou água de maceração de milho. É um resíduo que contém grandes quantidades de nutrientes, matéria orgânica e nitrogênio, o que possibilita sua reutilização como enriquecedor do meio fermentativo (LIGGETT et al., 1998). Avaliando o aspecto nutricional e econômico, além do caráter ambiental intrínseco ao processo, as características citadas demonstram a possibilidade da utilização destes dois resíduos para obtenção de ácido propiônico por via biológica. Porém, é necessário definir as condições que otimizem o processo, resultando em uma maior eficiência, a fim de possibilitar uma produção a nível industrial. Desta forma, o objetivo desse trabalho foi avaliar as melhores condições de concentração de lactose e água de maceração de milho para produção de ácido propiônico.

\section{MATERIAIS E MÉTODOS}

\subsection{Matérias-primas}

O soro ácido foi doado pelo laticínio Verde Campo, Lavras, MG e foi transportado imediatamente para o Laboratório de Engenharia de Bioprocessos (DCA 15), da Universidade Federal de Lavras, onde foi congelado em garrafas PET de $2 \mathrm{~L}$ até o momento das análises. Os microrganismos, Propionibacterium acidipropionici e Lactobacillus paracasei, foram adquiridos junto à Coleção de Culturas Tropical pertencente à Fundação André Tosello, de Campinas/SP.

\subsection{Preparo do meio de fermentação}

Preparação do soro e do inóculo: O soro foi filtrado a vácuo utilizando um sistema com kitasato, filtro qualitativo e bomba a vácuo (Vulkan 2 estágios 7 CFM VP200), para a retirada das micelas e homogeneização da matéria-prima. Foi realizada a concentração do soro utilizando um evaporador rotativo TE-2010. O soro foi concentrado e em seguida teve seu $\mathrm{pH}$ ajustado para 7, utilizando $\mathrm{NaOH} 0,01 \mathrm{~N}$, antes de ser submetido ao processo de esterilização em autoclave a $121^{\circ} \mathrm{C}$ por 15 minutos. O inóculo foi preparado a partir de uma cultura-estoque em glicerol, onde a cultura contida em micro tubo do tipo Eppendorf foi transferida para um tubo de ensaio contendo $10 \mathrm{~mL}$ de caldo lactato esterilizado. A cultura foi incubada a $30^{\circ} \mathrm{C}$ por $48 \mathrm{~h}$ e em seguida, transferida para um Erlenmeyer, com capacidade de $500 \mathrm{~mL}$, contendo $200 \mathrm{~mL}$ de meio com caldo lactato previamente esterilizado a $121^{\circ} \mathrm{C}$ durante 15 minutos. Após esse procedimento, a cultura foi incubada a $37^{\circ} \mathrm{C}$ durante 24 horas. As culturas assim obtidas foram padronizadas para a fermentação com base na correlação 


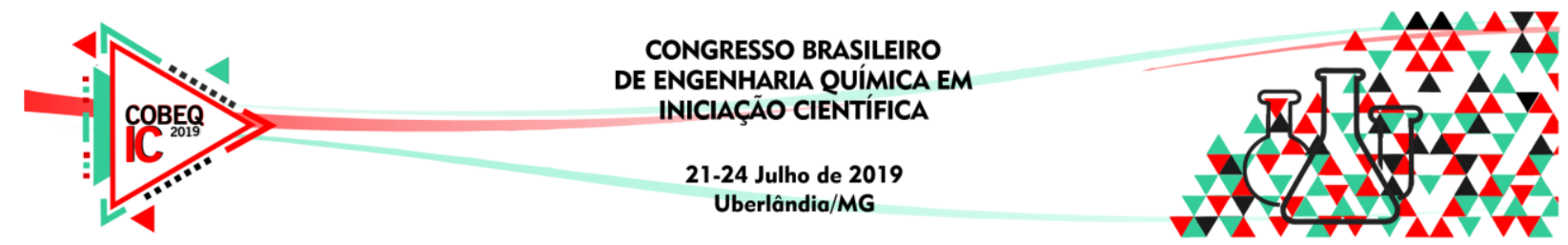

entre absorbância e o número de células viáveis através da curva de crescimento, correspondente a uma contagem de $10^{8} \mathrm{UFC} \mathrm{mL}^{-1}$. Posteriormente foram retiradas alíquotas deste inóculo, que correspondia a 10\% do meio, e inoculado em cada meio de fermentação.

Meios de cultura e condições de crescimento: A cultura de Propionibacterium acidipropionici NRRL B-3569 ativada foi inserida nos meios fermentados contendo $300 \mathrm{~mL}$ de meio composto de carbonato de cálcio, a uma concentração de $40 \mathrm{~g} / \mathrm{L}$, soro de leite ácido concentrado e água de maceração de milho em diferentes concentrações. A fermentação foi conduzida em Erlenmeyers, em incubadora SHAKER refrigerada (Solab, modelo SL-223), sem agitação, a $37^{\circ} \mathrm{C}$, com duração de $120 \mathrm{~h}$, sendo retiradas amostras de $15 \mathrm{~mL}$ de meio a cada $24 \mathrm{~h}$, que posteriormente foram centrifugadas e os sobrenadantes congelados para análises cromatográficas. Além disso, nos tempos de $0 \mathrm{~h}$ e $120 \mathrm{~h}$ foram retiradas amostras do meio de fermentação para avaliar o crescimento celular por meio de plaqueamento em superfície.

Foi realizado delineamento composto central rotacional (DCCR) com as variáveis concentração de lactose (x1) e concentração de água de maceração de milho (x2), conforme metodologia preconizada por RODRIGUES \& IEMMA (2012). A faixa de variação das variáveis independentes encontra-se na Tabela 1. As variáveis respostas são a produção do ácido propiônico e a contagem total das bactérias.

TABELA 1- Relação entre valores codificados e reais das variáveis independentes do DCCR

\begin{tabular}{cccccc}
\hline Variáveis & $\mathbf{- 1 , 4 1}$ & $\mathbf{- 1}$ & $\mathbf{0}$ & $\mathbf{1}$ & $\mathbf{1 , 4 1}$ \\
\hline $\mathbf{x 1}(\mathbf{C}$ lactose $-\mathbf{g} / \mathbf{L})$ & 26 & 30 & 40 & 50 & 54 \\
$\mathbf{x 2}(\mathbf{C}$ AMM $-\mathbf{g} / \mathbf{L})$ & 15 & 25 & 40 & 55 & 65 \\
\hline
\end{tabular}

\subsection{Métodos analíticos}

Organismos viáveis: Para a determinação dos organismos viáveis foi utilizado o método de plaqueamento em superfície em caldo ágar lactato, proposto por HARRIGAN \& MC CANCE (1976). As placas foram incubadas em jarras de anaerobiose, posteriormente deixadas em estufa microbiológica a $37^{\circ} \mathrm{C}$ por 120 horas. $\mathrm{O}$ fator de crescimento celular (FC) foi determinado utilizando a Equação 1:

$$
F C=\frac{C \text { final }}{C \text { inicial }}
$$

Sendo "C" o número de células viáveis totais em $\log \mathrm{UFC} / \mathrm{mL}$.

Determinação dos ácidos orgânicos: Os ácidos orgânicos foram identificados e quantificados por Cromatografia Líquida de Alta Frequência (CLAE), em cromatógrafo Shimadzu, com detector de condutividade $\left(\mathrm{CDD}-6^{\mathrm{a}}\right)$, polaridade + , utilizando uma pré coluna SHIM-PACK SPR-H(G) (50 mm x 7,8 mm), conforme metodologia adotada por NGOME et al. (2017).

Os parâmetros da produção foram determinados pelas Equações 2, 3 e 4: 


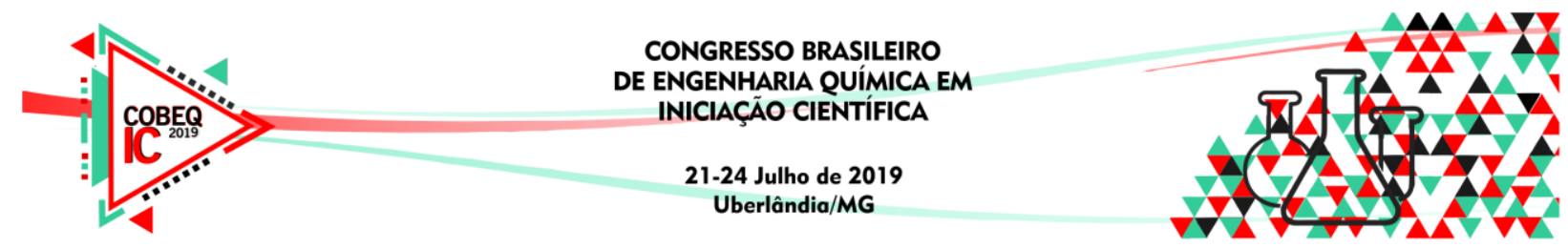

$$
\begin{aligned}
& \text { PAP }=[\text { AP }] \text { final }-[\text { AP }] \text { inicial } \\
& \text { PAL }=[\text { AL }] \text { final }-[\text { AL }] \text { inicial } \\
& \text { PAA }=[\text { AA }] \text { final }-[\text { AA }] \text { inicial }
\end{aligned}
$$

Sendo "PAP" a produção de ácido propiônico em g/L, "[AP]" a concentração de ácido propiônico em g/L, "PAL" a produção de ácido lático em g/L, "[AL]" a concentração de ácido lático em g/L, "PAA" a produção de ácido acético em g/L e "[AA]" a concentração de ácido acético.

Análise estatística: A análise estatística dos resultados obtidos foram submetidos à análise de variância, avaliação de efeitos ( $\mathrm{p}$-valor < 0,05) e avaliação da regressão utilizando pacote estatístico Statistica v. 8.0.

\section{RESULTADOS E DISCUSSÕES}

Na Tabela 2, são apresentados os resultados do DCCR.

TABELA 2 - Resultados do DCCR

\begin{tabular}{ccccccc}
\hline Trat. & $\mathbf{x 1}(\mathbf{g} / \mathbf{L})$ & $\mathbf{x 2}(\mathbf{g} / \mathbf{L})$ & $\mathbf{P A P}(\mathbf{g} / \mathbf{L})$ & $\mathbf{P A L}(\mathbf{g} / \mathbf{L})$ & $\mathbf{P A A}(\mathbf{g} / \mathbf{L})$ & $\mathbf{F C}$ \\
\hline $\mathbf{1}$ & 1 & 1 & 6,23 & 35,24 & 0,26 & 1,27 \\
$\mathbf{2}$ & -1 & 1 & 1,77 & 18,41 & 2,05 & 1,17 \\
$\mathbf{3}$ & 1 & -1 & 1,67 & 31,81 & 2,22 & 1,23 \\
$\mathbf{4}$ & -1 & -1 & 3,62 & 15,16 & 3,24 & 1,27 \\
$\mathbf{5}$ & 0 & 0 & 1,50 & 26,25 & 1,62 & 1,42 \\
$\mathbf{6}$ & 0 & 0 & 1,77 & 24,93 & 2,11 & 1,34 \\
$\mathbf{7}$ & 0 & 0 & 1,51 & 25,83 & 1,88 & 1,50 \\
$\mathbf{8}$ & 0 & 0 & 1,87 & 24,12 & 3,00 & 1,40 \\
$\mathbf{9}$ & $-1,41$ & 0 & 2,76 & 14,33 & 2,28 & 1,14 \\
$\mathbf{1 0}$ & 1,41 & 0 & 2,60 & 34,87 & 1,12 & 1,30 \\
$\mathbf{1 1}$ & 0 & $-1,41$ & 2,62 & 22,61 & 2,42 & 1,26 \\
$\mathbf{1 2}$ & 0 & 1,41 & 6,84 & 26,69 & 1,67 & 1,28 \\
\hline
\end{tabular}

Trat. - tratamento, x1 - C lactose, x2 - C AMM, PAP - Produção de ácido de propiônico, PAL - Produção de ácido lático, PAA - Produção de ácido acético e FC - Fator de crescimento

Pode-se observar na Tabela 2 que as produções de ácido propiônico, lático e acético variaram de 1,50 g/L (tratamento 5) a 6,84 g/L (tratamento 12), de 14,33 g/L (tratamento 9) a $35,24 \mathrm{~g} / \mathrm{L}$ (tratamento 1) e de 0,26 g/L (tratamento 1) a 3,24 g/L (tratamento 4), respectivamente. Verifica-se que houve efeitos estatisticamente significativos nas variáveis dependentes, para um nível de significância de 5\% ( $<<0,05)$ em alguns efeitos analisados, que estão apresentados na Tabela 3. Isto revela que as produções dos ácidos foram afetadas pela concentração de lactose e AMM. Pode-se observar que a produção de ácido propiônico foi afetada pela concentração de água de maceração e a produção de ácido lático, acético e o FC foram afetados pelas duas variáveis. 


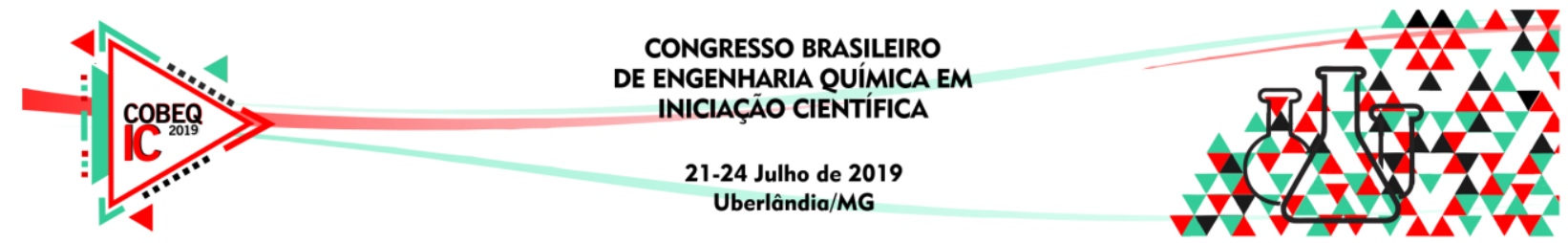

TABELA 3 - Coeficientes da regressão para produção de ácido propiônico (PAP), lático (PAL) e acético (PAA) após $120 \mathrm{~h}$ e do fator de crescimento (FC)

\begin{tabular}{|c|c|c|c|c|c|c|c|c|}
\hline \multirow[b]{2}{*}{ Fatores } & \multicolumn{2}{|c|}{ PAP } & \multicolumn{2}{|c|}{ PAL } & \multicolumn{2}{|c|}{ PAA } & \multicolumn{2}{|c|}{ FC } \\
\hline & Efeitos & $\mathbf{p}$ & Efeitos & $\mathbf{p}$ & Efeitos & $\mathbf{p}$ & Efeitos & $\mathbf{p}$ \\
\hline Média & 1,66 & 0,00 & 25,28 & 0,00 & 2,15 & 0,00 & 1,41 & 0,00 \\
\hline$(1) \operatorname{Lac}(g / L)(\mathbf{L})$ & 0,28 & 0,27 & 7,82 & 0,00 & $-0,55$ & 0,02 & 0,03 & 0,08 \\
\hline $\operatorname{Lac}(g / L)(\mathbf{Q})$ & 0,41 & 0,17 & $-0,20$ & 0,61 & $-0,21$ & 0,36 & $-0,10$ & 0,00 \\
\hline (2)AMM (g/L)(L) & 1,08 & 0,00 & 1,55 & 0,00 & $-0,52$ & 0,03 & 0,00 & 0,85 \\
\hline $\operatorname{AMM}(\mathbf{g} / \mathbf{L})(\mathbf{Q})$ & 1,44 & 0,00 & $-0,18$ & 0,65 & $-0,03$ & 0,87 & $-0,07$ & 0,01 \\
\hline $1 \mathrm{~L}$ by $2 \mathrm{~L}$ & 1,60 & 0,00 & 0,04 & 0,92 & $-0,19$ & 0,50 & 0,03 & 0,22 \\
\hline
\end{tabular}

$\mathrm{O}$ teste de Fisher mostrou que o ajuste da regressão é adequado, uma vez que $\mathrm{F}$ calculado é maior que o $\mathrm{F}$ tabelado, para as produções dos ácidos propiônico e lático e para o FC, mas não para a produção de ácido acético. Portanto plotou-se somente as curvas de contorno para produções de ácido propiônico e lático e o FC, mostrados na Figura 1.

Figura 1 - Influência da concentração de lactose e água de maceração de milho na produção de ácido propiônico (a), ácido lático (b) e FC (c).

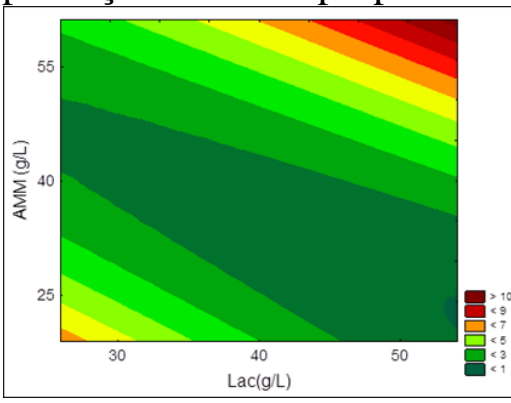

(a)

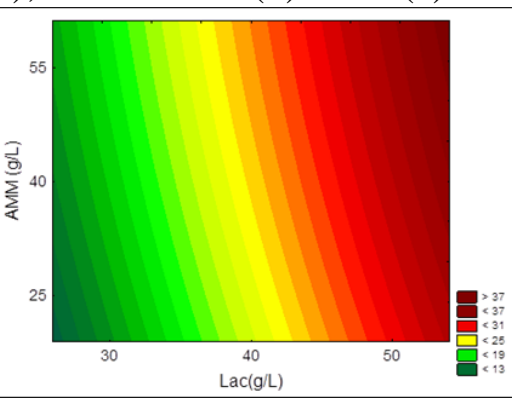

(b)

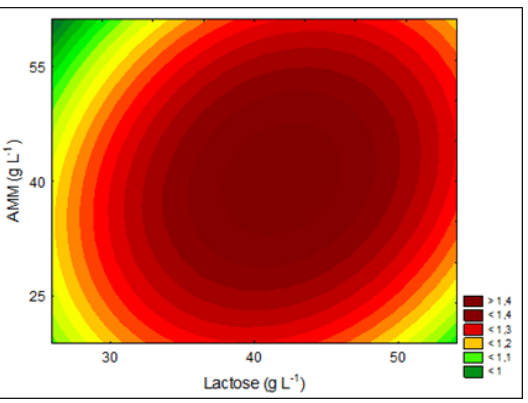

(c)

O tratamento 12, com concentrações de $40 \mathrm{~g} / \mathrm{L}$ de lactose e $65 \mathrm{~g} / \mathrm{L}$ de $\mathrm{AMM}$, foi o que apresentou a maior produção de ácido propiônico $(6,84 \mathrm{~g} / \mathrm{L})$. O valor encontrado é similar aos obtidos em alguns trabalhos publicados. Experimentos realizados por TELES (2016) apresentaram 0,40 g/L, utilizando somente a Propionibacterium acidipropionici, porém com outro substrato. Os resultados NGOME et al. (2017) apresentram produção de 3,78 g/L, utilizando Propionibacterium freudenreichii para fermentar soro de leite e AMM. A curva de contorno para produção de ácido propiônico apresentada na Figura 1(a) mostra que concentrações acima de $50 \mathrm{~g} / \mathrm{L}$ de $\mathrm{AMM}$ e acima de $45 \mathrm{~g} / \mathrm{L}$ de lactose favorecem a sua produção.

A curva de contorno para produção de ácido lático, ilustrada na Figura 1(b), mostra que para maiores concentrações de lactose, acima de $45 \mathrm{~g} / \mathrm{L}$, há maior produção de ácido lático. $\mathrm{O}$ aumento da concentração de $\mathrm{AMM}$ tem efeito positivo, mas com menor influência sobre produção de ácido lático. A curva de contorno para o fator de crescimento, apresentada na Figura 1(c), mostra que há maior crescimento das bactérias em concentrações próximas de $40 \mathrm{~g} / \mathrm{L}$, tanto para AMM, como para lactose. 


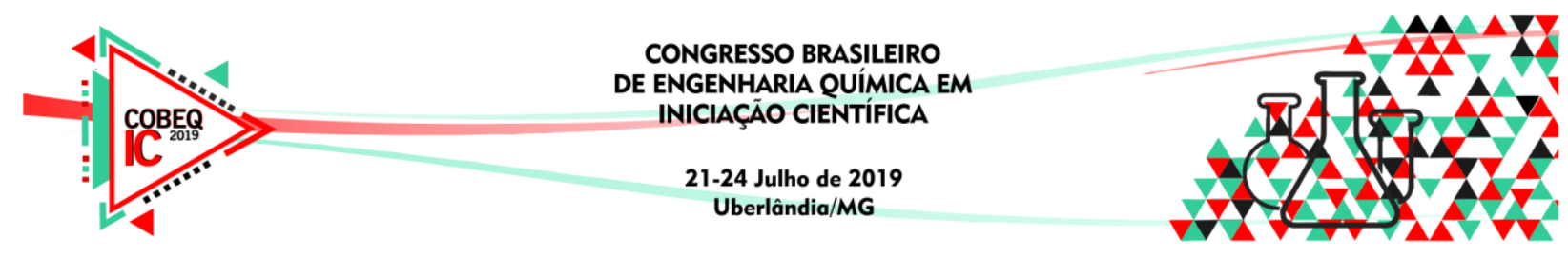

\section{CONCLUSÃO}

De acordo com variáveis resposta, pode-se observar que houve maior eficiência do processo quando o meio de fermentação apresentou concentrações de lactose maiores que 50 $\mathrm{g} / \mathrm{L}$ e concentrações de água de maceração de milho acima de $45 \mathrm{~g} / \mathrm{L}$, pois houve maior produção de ácido propiônico. O tratamento 12 foi o que apresentou a maior concentração de ácido propiônico $(6,84 \mathrm{~g} / \mathrm{L})$.

\section{REFERÊNCIAS}

ATRA, R. et al. Investigation of ultra and nanofiltration for utilization of whey protein and lactose. Journal of Food Engineering, v. 67, n. 3, p. 325-332, 2005.

BALDASSO, C. Concentração, purificação e fracionamento das proteínas do soro lácteo através da tecnologia de separação por membranas. 2008. 163 p. Dissertação (Mestrado em Engenharia) - Universidade Federal do Rio Grande do Sul, Escola de Engenharia, Porto Alegre, 2008.

CHEN, F. et al. Propionic acid production in a plant fibrous-bed bioreactor with immobilized Propionibacterium freudenreichii CCTCC M207015. Journal of Biotechnology, v. 164, n. 2, p. 202-210, 2012.

DISHISHA, T.; ALVAREZ, M. T.; HATTI-KAUL, R. Batch- and continuous propionic acid production from glycerol using free and immobilized cells of Propionibacterium acidipropionici. Bioresource Technology, v. 118, p. 553-562, 2012.

HARRIGAN, Wilkie Francis et al. Laboratory methods in food and dairy microbiology. Academic Press Inc.(London) Ltd., 1976.

LIGGET, W. R; KOFFLER H. Corn steep liquor in microbiology. Purdue University, v.12, U.S.A. 1998.

NGOME, M. T.; ALVES , J. G. L. F.; PICCOLI R. H; DOMINGO, E. C; PINTO, S. A; MONDRAGÓN BERNAL, O. L; Inoculum concentration and inoculation time for propionic acid production from whey using mixed culture of Lactobacillus helveticus and Propionibacterium freudenreichii PS-1. Acta Scientiarum. Technology, v. 39, p. 543-550, 2017.

RODRIGUES, Maria Isabel; IEMMA, Antonio Francisco. Experimental design and process optimization. CRC Press, 2014.

SABRA, W.; DIETZ, D.; ZENG, A. P. Substrate-limited co-culture for efficient production of propionic acid from flour hydrolysate. Applied Microbiology and Biotechnology, v. 97, n. 13, p. 5771-5777, 2013.

TELES, Jéssica Cristine. Produção de ácido propiônico por Propionibacterium acidipropionici a partir da fermentação de efluentes agroindustriais. 2016. $62 \mathrm{f}$. Dissertação (Mestrado em Ciências e Tecnologia de Alimentos) - UNIVERSIDADE ESTADUAL DE PONTA GROSSA, Ponta Grossa, 2016. 